\section{Comparison of perceptions on the dental aesthetics of different malocclusions between orthodontists and schoolchildren}

\author{
Bowen Zheng, MD, Fenik K. Muhammed, MSc, PhD, \\ Na An, MD, Lu Bai, MD, Fan Liu, MD, Ying Zheng, MD,
} Yi Liu, MD, PhD.

\section{ABSTRACT}

Objectives: To compare perceptions on dental aesthetics of various malocclusions between schoolchildren and orthodontists with an orthodontic assessment of treatment needs.

Methods: The prospective study was conducted between late March-December in 2017. A total of 32 orthodontists practicing in Shenyang, China and 116 schoolchildren (59 boys and 57 girls) aged $10-12$ years (average $=11 \pm 0.60$ years) from a 645 individual representing the population were selected via random sampling from a private primary school. Participants were questioned to assess 6 anterior dental photographs representing a variety degrees of aesthetic impairments, namely, reverse overjet (ROJ), anterior dental diastema and anterior open bite (OB) malocclusions.

Results: Perceptions on dental aesthetics were identical among genders $(p>0.05)$. Compared with the orthodontists, schoolchildren perceived significantly greater aesthetic impairment for severe anterior $\mathrm{OB}$ and severe $\mathrm{ROJ}$, mild anterior $\mathrm{OB}$ malocclusions $(p<0.01)$. Anterior dental diastemas were evaluated based on least aesthetic impairment and with no significant difference in perception between schoolchildren and orthodontists.

Conclusion: Schoolchildren perceived greater aesthetic impairment in severe anterior $\mathrm{OB}$, severe $\mathrm{ROJ}$, and mild anterior $\mathrm{OB}$ malocclusions than orthodontists. Median aesthetic components (AC) scores of schoolchildren for severe anterior $\mathrm{OB}$ and severe ROJ malocclusions corresponded with the category of "definite need" for treatment.

Saudi Med J 2018; Vol. 39 (9): 946-950 doi: 10.15537/smj.2018.9.23482

$\mathrm{O}$ rthodontic indexes have been established to standardize orthodontic care assessment. The cosmetic impairment of a particular malocclusion is challenging to measure and identify because of the highly subjective perception towards malocclusion. Several authors have described the criteria for an occlusal index. ${ }^{1,2}$ The index of orthodontic treatment need (IOTN) is a method by Shaw et al, ${ }^{3,4}$ for assessing aesthetic impairments in malocclusions and determining the need for orthodontic treatment. The index of orthodontic treatment need incorporates 2 independent components: the dental health component (DHC) and AC. The dental health component determines the need for treatment on the basis of dental health with its functional aspects and involves 5 grades. Meanwhile, AC can assess individual viewpoints on dental attractiveness and judge the appropriate treatment. ${ }^{5,6}$ The aesthetic components is considered reflective of aesthetic impairment and is composed of a scale of 10 coloured intraoral photographs illustrating various malocclusion levels, from most attractive to least attractive. ${ }^{6}$

Reverse overjet appears when the upper incisors lie in lingo-occlusion with respect to the lower anterior teeth. The term anterior crossbite is commonly used when only one or 2 incisors are involved.'

A diastema can be defined as the presence of a space between 2 teeth. This abnormality can be treated with orthodontics or cosmetic dentistry. ${ }^{7}$

An anterior open bite $(\mathrm{OB})$ is a vertical gap between the maxillary and mandibular incisors with teeth in maximum intercuspation. ${ }^{8}$ Anterior $\mathrm{OB}$ can be classified as mild or severe; the former can be treated orthodontically, whereas the latter may entail significant vertical skeletal component combined with orthodontic-surgical treatment. ${ }^{8}$

Methods. This study was approved by the Local Research Ethics Committee of Stomatology of China Medical University in Shenyang, China. All procedures followed were in accordance with the principles of Helsinki Declaration. The prospective study was conducted between late March-December in 2017. A total of 116 schoolchildren (59 boys and 57 girls) were selected by simple random sampling from a 645individual representative population in a private primary school in Shenyang, China. The average age of subjects was $11 \pm 0.60(10-12)$ years. The subjects with previous or ongoing orthodontic therapy were excluded from the study. The schoolchildren in this age group are in the late mixed dentition, the time at which orthodontic

Disclosure. This study was funded by Liaoning Province Science Enterprise Commonweal Research Fund Project (2015005003) from China. 
treatment begins to be considered. ${ }^{9}$ Informed consent forms were sent to the participants' homes one week before study commencement. The schoolchildren who were given informed consent by their parents completed a 45-minute confidential questionnaire. Meanwhile, 32 orthodontists with 5 years' clinical experience were selected from the Stomatology Hospital, China Medical University, Shenyang, China, to participate in this study.

A data collection sheet was prepared for the subjects and used to record each participant's age in years and months, gender and perceptions on dental aesthetics. ${ }^{10}$ The study was undertaken during school hours in a well-lit room. The subjects recorded their gender, age and perceptions on dental aesthetics in the data collection sheet. As tools for measuring the perceptions on dental aesthetics ${ }^{10}$ frontal intraoral photographs were progressively ordered from $1-10$ in 2 columns representing the $\mathrm{AC}$ of the IOTN (Figure 1). ${ }^{10}$

The AC-IOTN scores were ranked from the most positive dental appearance (most attractive) to the most negative dental appearance (least attractive). ${ }^{11}$ Six separately lettered (A-F) anterior photographs (Figure 2) of 3 malocclusions, including ROJ, dental diastema and anterior $\mathrm{OB}$ with their mild and severe degrees, were collected from patient data from the Department of Orthodontics, Stomatology Hospital, China Medical University, Shenyang, China by the principal author.

A PowerPoint 2013 presentation (Microsoft, Redmond, Washington, USA) comprising 6 slides was prepared. Each slide displayed one of the 6 lettered photos. The AC of the IOTN was projected as a coloured slide on a $2 \mathrm{~m} \times 2 \mathrm{~m}$ white screen in a schoolroom while the participants answered the questionnaire.

The subjects were initially asked to study all the 10 photographs of the AC of IOTN and note that photo one represented the most attractive set of teeth and photograph 10 as the least attractive. The participants were then instructed to assess the lettered photographs on the PowerPoint slide based on the reference images of the 10-point scale AC-IOTN. Their scores were recorded on the data collection sheet. Sharing of opinions between peers or teachers was not
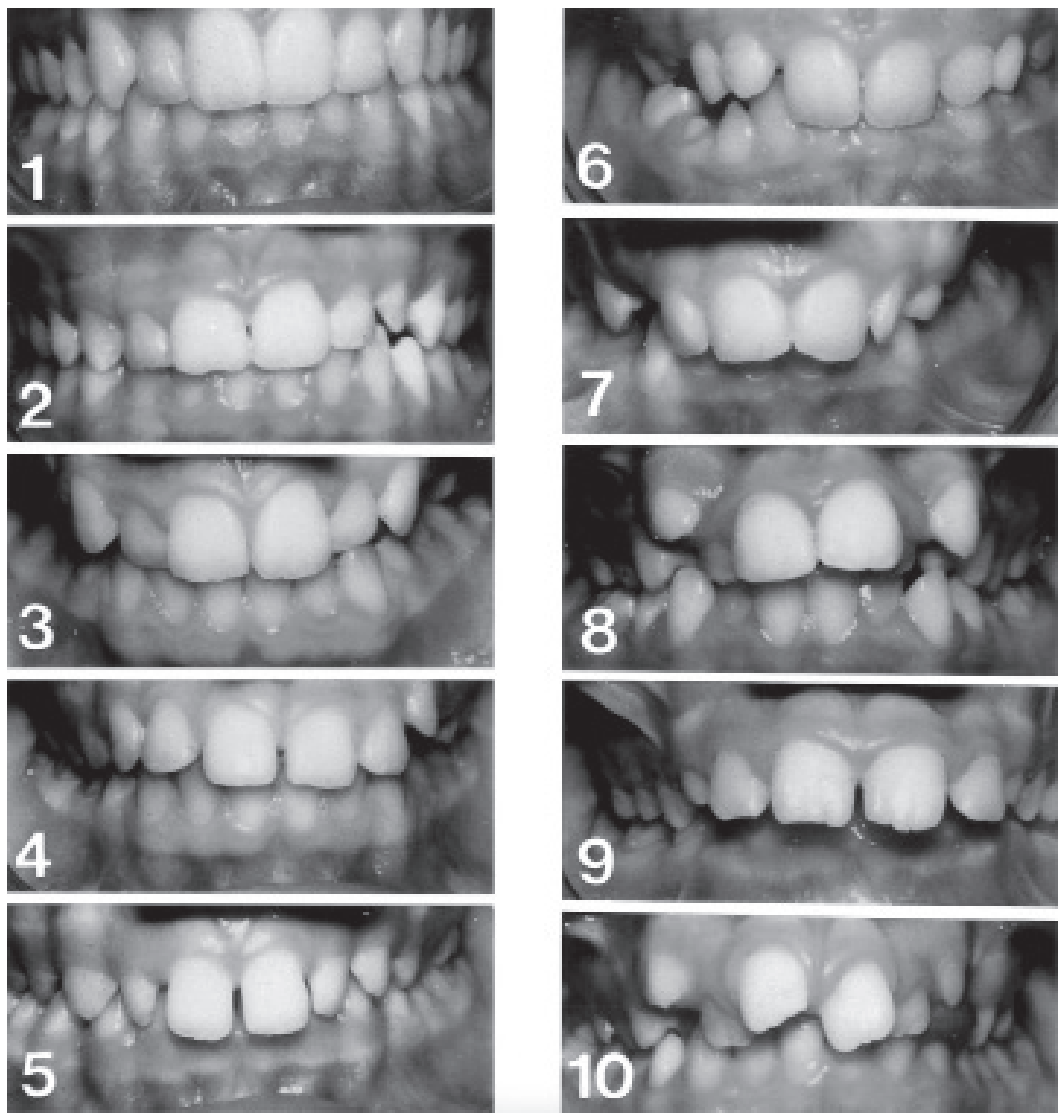

Figure 1 - The aesthetic component of the index of orthodontic treatment need (IOTN). ${ }^{10}$

Reprinted from The European Journal of Orthodontics, 75/6, Singh V, Hamdan A, Rock W, The perception of dental aesthetics and orthodontic treatment need by 10 - to 11-year-old children, Copyright 4387621273900, with permission from Copyright Clearance Center 


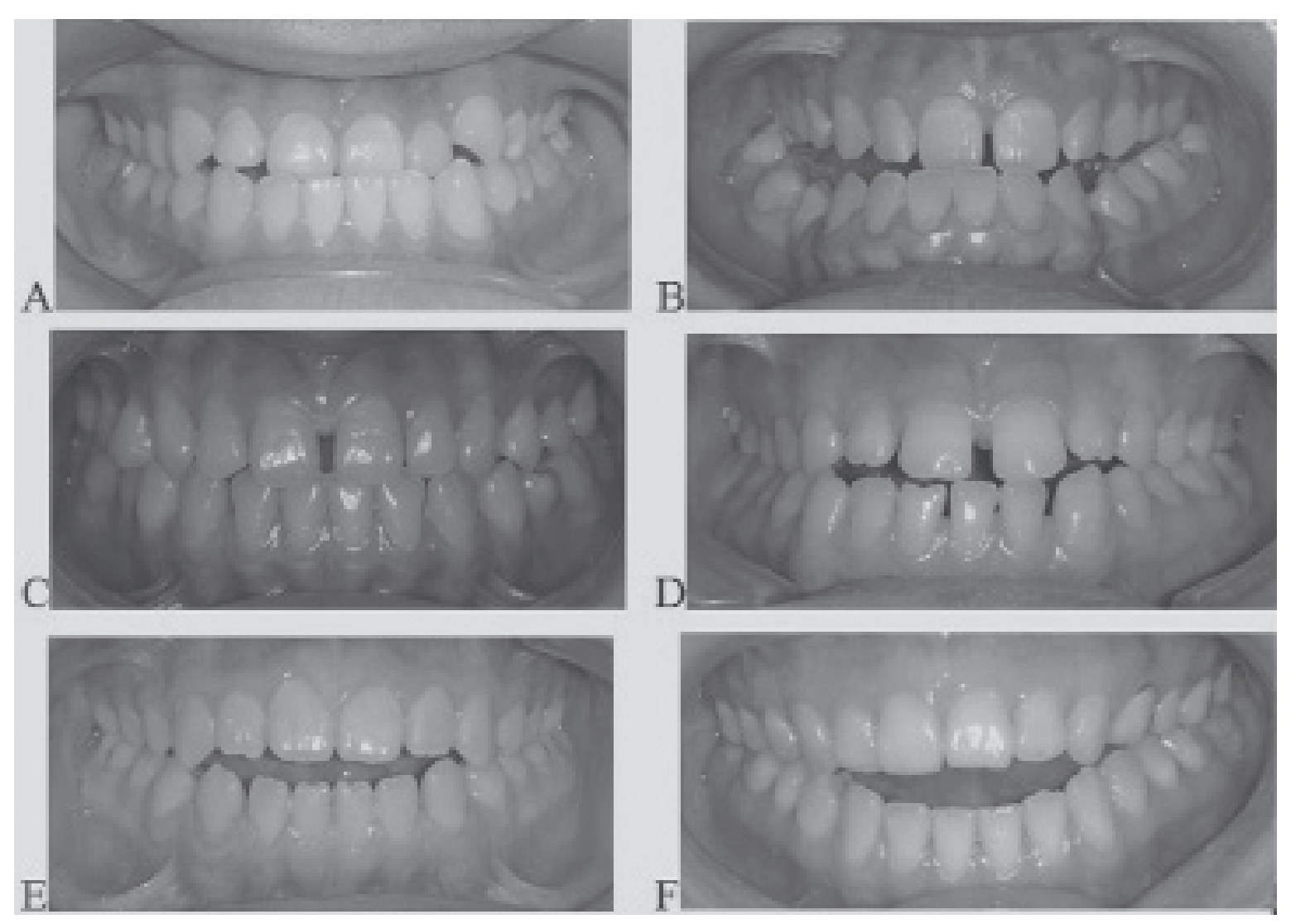

Figure 2 - The lettered photographs of malocclusion of varying severity. A) Mild anterior reverse overjet (ROJ). B) Sever anterior reverse overjet (ROJ). C) Mild anterior dental diastema D) Sever anterior dental diastema. E) Mild anterior open bite (OB). F) Sever anterior open bite $(\mathrm{OB})$.

allowed during the exam. No details were given to the participants regarding the photographs. Data collection from orthodontists was carried out in a lecture room by the principal author under exam conditions similar to those with the schoolchildren.

Statistical analysis. Statistical analysis was carried out with SPSS $17.0^{\circledR}$ (SPSS Inc., Chicago, IL, USA). Moreover, a multivariable general linear model was used to evaluate the influence of gender, subject group and photos on the AC scores. Furthermore, the MannWhitney test was applied for each photo comparison. A $p$-value $<0.05$ was considered significant.

Results. Table 1 shows the schoolchildren's perceptions on dental aesthetics, with the AC of IOTN as basis. Both genders allocated the highest median AC scores to severe anterior $\mathrm{OB}$ and severe ROJ, which are represented by photos $\mathrm{F}$ and $\mathrm{B}$. The boys assigned similar median AC scores to photos D and E, which corresponded to severe dental diastema and mild anterior $\mathrm{OB}$; and ascribed the lowest median AC score to mild ROJ and mild dental diastema. According to multivariable statistical analysis, gender (schoolchildren and orthodontists) was not a significant variable $(p>0.05)$.

Table 2 illustrates the perceptions of schoolchildren and orthodontists towards dental aesthetics. According to multivariate statistical analysis, the subject group and photos were significant at $(p<0.05)$ and $(p<0.01)$. Moreover, Mann-Whitney test was applied to individual photographs. Schoolchildren and orthodontists allocated the highest median $\mathrm{AC}$ scores to photos $\mathrm{F}$ and $B$, which represents severe anterior $\mathrm{OB}$ and severe ROJ. The scores were highly and significantly greater amongst the schoolchildren than amongst the orthodontists $(p<0.01)$.

Similarly, the schoolchildren's scores for photo E, which denoted mild anterior $\mathrm{OB}$ was significantly $(p<0.01)$ greater than those of the orthodontists. By contrast, the lowest median AC score was assigned by both groups to photo $\mathrm{C}$, which corresponded to mild dental diastema, and the score differences were insignificant $(p>0.05)$. 
Discussion. The present study was conducted on 116 schoolchildren aged 10-12 years from a private primary school in Shenyang, China. Several authors have reported that children younger than 10 years find difficulty in judging aesthetic improvement. ${ }^{10,12}$ To compare the perceptions on dental aesthetics, our study selected the evaluation of the mild and severe degrees of malocclusion ROJ, anterior dental diastema and anterior $\mathrm{OB}$. Anterior dental spacing was included under the malocclusal trait AC of the IOTN, whereas ROJ and anterior $\mathrm{OB}$ were not included in the 10 photographs of the IOTN. ${ }^{8}$ The perceptions on dental aesthetics were identical amongst girls and boys. This finding is consistent with previous studies. ${ }^{10,12-14}$ However, other authors showed gender differences, females being more concerned with dental aesthetics than males. ${ }^{12,15}$

In the present study, the schoolchildren and orthodontists perceived greater aesthetic impairment in severe anterior $\mathrm{OB}$ and severe $\mathrm{ROJ}$ malocclusions than in mild dental diastema. The former malocclusion images were assigned with higher median AC scores than those of the latter malocclusion. Severe anterior $\mathrm{OB}$ and severe ROJ malocclusions exerted a greater impact than that of mild and severe dental diastema on the perception of aesthetics in both groups. This finding agrees with those of Hamdan et $\mathrm{al},{ }^{10}$ who observed that moderate and severe Class III malocclusions achieved a greater effect than those of other conditions on the perceptions of aesthetics amongst schoolchildren and orthodontists. The perception of aesthetic impairment in severe anterior $\mathrm{OB}$ and severe $\mathrm{ROJ}$ malocclusions was worse amongst the schoolchildren than amongst the orthodontists. A previous work investigated attractiveness ratings based on AC of IOTN and reported that severe ROJ were assessed identically by dental professionals and non-dental students, but the perception differences of the severe anterior OB were significant. ${ }^{8}$ Schoolchildren and orthodontists hold similar perceptions toward mild and severe dental diastema. A previous study that applied the AC of IOTN reported that clinicians' ratings were more critical than those of children or laymen. ${ }^{13}$

The cut-off points for no, borderline, and definite need of treatment were introduced by the dental professional opinion as a gold standard validation of the AC. Photos one-four represent no/slight treatment need; 5-7 represent moderate/borderline need, and 8-10 represent definite treatment need (Figure 1). ${ }^{10}$ The median AC scores of severe anterior $\mathrm{OB}$ and severe $\mathrm{ROJ}$ were assigned by the schoolchildren to the 5 photos in the category of 'Definite treatment need'. A previous study has found that schoolchildren experience difficulty assessing aesthetic impairments in photos representing severe anterior $\mathrm{OB}$ and severe $\mathrm{ROJ}$ malocclusions. The

Table 1 - Schoolchildren perceptions of dental aesthetics using the aesthetic component (AC) of index of orthodontic treatment need (IOTN).

\begin{tabular}{|c|c|c|c|c|c|c|}
\hline Photograph letters & Photograph & Boys & $n=59$ & Gir & $\mathrm{s} \mathbf{n}=57$ & $P$-value \\
\hline & & Median & Mean \pm SD & Median & Mean \pm SD & \\
\hline $\mathrm{A}$ & Mild ROJ & 3.0 & $3.6 \pm 1.98$ & 3.0 & $3.8 \pm 1.89$ & 0.575 \\
\hline B & Severe ROJ & 8.0 & $6.9 \pm 1.83$ & 8.0 & $6.2 \pm 1.91$ & 0.132 \\
\hline $\mathrm{C}$ & Mild dental diastema & 3.0 & $3.4 \pm 1.96$ & 3.0 & $3.0 \pm 2.17$ & 0.745 \\
\hline $\mathrm{D}$ & Severe dental diastema & 4.0 & $5.0 \pm 1.94$ & 5.0 & $5.6 \pm 1.83$ & 0.053 \\
\hline $\mathrm{E}$ & Mild anterior $\mathrm{OB}$ & 4.0 & $4.2 \pm 2.68$ & 4.0 & $4.2 \pm 2.25$ & 0.345 \\
\hline $\mathrm{F}$ & Severe anterior OB & 8.5 & $7.9 \pm 2.25$ & 8.5 & $8.1 \pm 2.83$ & 0.516 \\
\hline
\end{tabular}

Table 2 - Comparison of schoolchildren's and orthodontist's perceptions of dental aesthetics.

\begin{tabular}{lcccccc}
\hline Photograph letters & Photograph & \multicolumn{2}{c}{ Schoolchildren $\mathrm{n}=116$} & Orthodontists $\mathrm{n}=32$ & $P$-value \\
\hline & & Median & Mean \pm SD & Median & Mean \pm SD \\
A & Mild ROJ & 3.5 & $3.9 \pm 1.65$ & 4 & $3.7 \pm 1.04$ & $0.03^{*}$ \\
B & Severe ROJ & 8 & $6.6 \pm 1.62$ & 7.5 & $5.4 \pm 1.60$ & $0.004^{* *}$ \\
C & Mild dental diastema & 3 & $3.1 \pm 1.08$ & 3 & $3.2 \pm 1.19$ & 0.596 \\
D & Severe dental diastema & 5 & $5.6 \pm 1.99$ & 5 & $5.0 \pm 1.55$ & 0.327 \\
E & Mild anterior OB & 4 & $5.4 \pm 1.95$ & 3 & $4.17 \pm 1.01$ & $0.009^{* *}$ \\
F & Severe anterior OB & 8.5 & $6.1 \pm 1.60$ & 7 & $5.9 \pm 1.71$ & $0.001^{* *}$ \\
\hline \multicolumn{7}{c}{} \\
\hline
\end{tabular}


median score was not assigned with a treatment need. ${ }^{10}$ Severe anterior OB, severe ROJ, and severe dental diastema malocclusions were allocated to the 'Borderline need' category by the orthodontists. The present findings seem to be consistent with other research which found severe anterior $\mathrm{OB}$ and severe $\mathrm{ROJ}$ malocclusions were allocated to the borderline need category by the dental professional opinion. ${ }^{10}$

The present study increases our knowledge of the schoolchildren's perception and assessment in treatment need of malocclusions not included in the AC of IOTN. It has been shown that both of severe anterior $\mathrm{OB}$ and severe ROJ malocclusions are in the level of sensitivity (identifying those that needed treatment) according to schoolchildren perceptions that has not been identified by the orthodontists. Schoolchildren's perception and assessment should be considered when formulating orthodontic treatment plans. Further research are needed to explore and identify the difference in perceptions of dental aesthetic between schoolchildren and orthodontics and factors that influence the decision of orthodontic treatment. These results can be generalised, but the generalization that can be drawn have certain limitations. For instance, the limited number of schoolchildren participants and the convenience sampling of the 32 orthodontists are the potential limiting factors. The authors exerted massive efforts to maximize the number of schoolchildren to be included, but some parents did not return their participation forms. Furthermore, the random sampling of participant orthodontists is more appropriate than that applied in this work.

In conclusion, Perceptions of dental aesthetics regarding malocclusions were similar between the male and female schoolchildren. By contrast, the schoolchildren perceived significantly greater impairment in the photos representing severe anterior $\mathrm{OB}$, severe $\mathrm{ROJ}$ and mild anterior $\mathrm{OB}$ malocclusions than that perceived by the orthodontists. Median AC scores of schoolchildren for severe anterior $\mathrm{OB}$ and severe ROJ malocclusions corresponded with the category of "definite need" for treatment. Therefore, for effective orthodontic care, schoolchildren's perception and assessment should be considered when formulating orthodontic treatment plans.

Acknowledgment. We would like to thank KG Support Limited (www.kgsupport.com) for English language editing of our manuscript. We would like to express our deep gratitude to Prof. Raad N. Dayem, for contributing this project.

Received 13th June 2018. Accepted 25th July 2018.

From the Department of Orthodontics, School of Stomatology, China Medical University, Shenyang, China. Address correspondence and reprints request to:
Prof. Yi Liu, Department of Orthodontics, School of Stomatology, China Medical University, Shenyang, China.E-mail: liuyi@cmu.edu.cn

ORCID ID: orcid.org/0000-0002-4640-3396

\section{References}

1. Bellot-Arcís C, Montiel-Company JM, Almerich-Silla JM. Psychosocial impact of malocclusion in Spanish adolescents. Korean J Orthod 2013; 43: 193-200.

2. Borzabadi-Farahani A. An insight into four orthodontic treatment need indices. Prog Orthod 2011; 12: 132-142.

3. Khanehmasjedi M, Bassir L, Haghighizade MH. Evaluation of orthodontic treatment needs using the dental aesthetic index in Iranian students. Iran Red Crescent Med J 2013; 15: e10536.

4. Ajayi EO. Normative and self-perceived orthodontic treatment need in Nigerian school children. Acta Odontol Scand 2015; 73: 364-367.

5. Puertes-Fernández N, Montiel-Company JM, AlmerichSilla JM, Manzanera D. Orthodontic treatment need in a 12-year-old population in the Western Sahara. Eur J Orthod 2011; 33: 377-380.

6. Borzabadi-Farahani A. Agreement between the index of complexity, outcome, and need and the dental and aesthetic components of the index of orthodontic treatment need. Am J Orthod Dentofacial Orthop 2011; 140: 233-238.

7. Mitchell L, editor. An introduction to orthodontics. Oxford (UK); Oxford University Press: 2013. p. 108-161.

8. Abu Alhaija ES, Al-Khateeb SN. Attractiveness ratings of anterior open bites and reverse overjets using the aesthetic component of the Index of Orthodontic Treatment Need. Eur J Orthod 2005; 27: 134-139.

9. Hamdan AM, Singh V, Rock W. Perceptions of dental aesthetics of Class III and anterior open bite malocclusions: a comparison between 10- to 11-year-old schoolchildren and orthodontists. Angle Orthod 2012; 82: 202-208.

10. Singh V, Hamdan A, Rock P. The perception of dental aesthetics and orthodontic treatment need by 10 - to 11-year-old children. Eur J Orthod 2012; 34: 646-651.

11. Salih FN, Lindsten R, Bågesund M. Perception of orthodontic treatment need among Swedish children, adolescents and young adults. Acta Odontol Scand 2017; 75: 407-412.

12. Hamdan AM, Singh V, Rock WP. Assessment of the relationship between perceptions of dental aesthetics and demand for orthodontic treatment in 10-11 year old school children in Birmingham, UK. Community Dent Health 2012; 29: 124-128.

13. Hamdan AM. The relationship between patient, parent and clinician perceived need and normative orthodontic treatment need. Eur J Orthod 2004; 26: 265-271.

14. Ngom PI, Diagne F, Dieye F, Diop-Ba K, Thiam F. Orthodontic treatment need and demand in Senegalese school children aged 12-13 years. An appraisal using IOTN and ICON. Angle Orthod 2007; 77: 323-330.

15. Christopherson EA, Briskie D, Inglehart MR. Objective, subjective, and self-assessment of preadolescent orthodontic treatment need--a function of age, gender, and ethnic/racial background? J Public Health Dent 2009; 69: 9-17. 